\title{
Dance at home for people with Parkinson's during COVID-19 and beyond: Participation, perceptions and prospects
}

\author{
Judith Bek ${ }^{1,2 *}$, Michelle Groves ${ }^{3}$, David Leventhal ${ }^{4}$, Ellen Poliakoff ${ }^{1}$ \\ ${ }^{1}$ Division of Neuroscience and Experimental Psychology, School of Biological Sciences, University of Manchester, UK \\ ${ }^{2}$ School of Psychology, College of Social Sciences and Law, University College Dublin, Ireland \\ ${ }^{3}$ Faculty of Education, Royal Academy of Dance, London, UK \\ ${ }^{4}$ Mark Morris Group - Dance for PD, Brooklyn, NY, USA
}

\begin{abstract}
Emerging evidence shows that dance can provide both physical and non-physical benefits for people living with Parkinson's disease (PD). The suspension of in-person dance classes during the COVID-19 pandemic necessitated a transition to remote provision via live and recorded digital media.

An online survey explored accessibility of and engagement with these home-based dance programs, as well as perceived benefits. The survey was co-developed by researchers and dance program providers, with input from people with PD and physiotherapists.

Responses were collected from 276 individuals, including 178 current users of home-based programs, the majority of whom were participating at least once per week. Among respondents not currently using digital resources, lack of knowledge and motivation were the primary barriers. Most participants (94.9\%) reported that home based practice provided some benefits, including physical (e.g., balance, posture) and non-physical (e.g., mood, confidence) improvements. Participants valued the convenience and flexibility of digital participation, but noted limitations including reductions in social interaction, support from instructors and peers, and routine. There was a strong preference $(70.8 \%)$ for continuing with home-based practice alongside in-person classes in the future.

The results indicate that at-home dance is accessible and usable for people with PD, and that some of the previously-reported benefits of dance may be replicated in this context. While COVID-19 expedited the development of digital programs, these will likely remain a key element of future provision for people with PD. The findings will inform the further development of resources and research into outcomes of home-based dance participation.
\end{abstract}

Keywords: Parkinson's disease; dance; home-based; digital health; COVID-19

\section{Introduction}

Parkinson's disease (PD) is one of the most prevalent and fastest growing neurological conditions (Dorsey et al., 2018), and is characterised by multiple motor and sensorimotor symptoms including rigidity, tremor, postural instability and disturbances in gait and balance, as well as bradykinesia (slowing of movement) and hypokinesia (reduced amplitude of movement). People with PD can also experience a wide range of non-motor symptoms, including cognitive, emotional and behavioural problems, pain and autonomic dysfunction (Chaudhuri et al., 2006).

The coronavirus disease COVID-19 has been associated with a deterioration in PD symptoms (Brown et al., 2020; Cilia et al., 2020), but the wider effects of the pandemic are also likely to have exacerbated the social isolation, apathy and anxiety already commonly experienced by people with PD (Helmich \& Bloem, 2020; Subramanian, 2020). Activities that may mitigate some of these 
effects by maintaining social connections, as well as physical and psychological wellbeing, are therefore likely to be of great value to people with PD.

Non-medical approaches including physiotherapy and exercise are widely recommended for people with PD (Keus et al., 2014), and dance is a multidimensional activity that offers an engaging, low-cost and sustainable therapeutic option. A substantial body of literature has studied various dance styles and genres for people with PD, and systematic reviews have highlighted potential benefits, particularly in terms of motor symptoms, gait, functional mobility and cognition (e.g., dos Santos Delabary et al., 2018; Sharp \& Hewitt, 2014). A number of dance programs have been developed specifically for older people and those with neurological conditions, including Silver Swans (Royal Academy of Dance, 2016 ${ }^{1}$ ) and Dance for PD (Mark Morris Dance Group, 20012), which both feature expanding networks of in-studio classes delivered by trained instructors worldwide.

The global spread of COVID-19 in early 2020 resulted in the sudden imposition of restrictions in activity and social contact, necessitating the abrupt suspension of group activities such as dance. Providers of community dance programs worked quickly to adapt to online delivery, transitioning to media such as video recordings, live streaming and interactive videoconferencing platforms. Recent research has indicated that people with $P D$ have been able to use technology-based tools to remain physically active during lockdown (Schirinzi et al., 2020), and positive engagement with remote delivery of individual physical activity coaching has been found (Quinn et al., 2020). However, the importance of the social aspects of dance in producing positive effects on well-being is often highlighted (e.g., Houston \& McGill, 2013; Kunkel et al., 2018), and while the potential value of virtual support groups has been suggested (Subramanian, 2020), little is known about how people with PD may engage with and benefit from online group activities.

Given the emerging evidence showing quantifiable effects of dance for people with PD, as well as its perceived role in maintaining well-being

\footnotetext{
${ }^{1}$ https://www.royalacademyofdance.org/classes/silverswans/
} 2 https://danceforparkinsons.org/ among participants, it is important to understand how people with PD access and utilise remotely delivered programs, and the potential physical and non-physical benefits of these programs, particularly in comparison with in-person group classes.

Beyond keeping participants engaged in activity and maintaining well-being through the COVID-19 pandemic, it is also important to consider how the experiences of this situation might shape future provision of dance programs even once in-person classes can be resumed. It is likely that digital classes and resources will change the landscape of community dance in the longer term, for several reasons: (i) restrictions on - or anxiety surrounding - group activities may remain for some time; (ii) a greater volume or variety of digital provision may lead to changes in user preferences, resulting in increased replacement or supplementation of in-person classes; (iii) digital programs could promote broader accessibility and uptake of dance by offering a flexible and convenient alternative, particularly for those who find it difficult to physically attend classes (e.g., because of geographical location or transport issues) or prefer to practice at home.

While there may be advantages to homebased dance programs, several potential limitations should also be considered. For example, the need for confidence to engage with digital technologies, or the ability to afford digital access, may present barriers to participation. Additionally, while engaging with at-home dance resources may provide increased opportunities for participation for some individuals, for others it may result in a loss of routine and reduced motivation. Moreover, the social aspects of dance may be difficult to replicate in virtual settings and pre-recorded sessions. The loss of the group dynamic and faceto-face interactions with the instructor and other dancers may impact not only on enjoyment of classes, but also on some of the physical, cognitive and psychological benefits associated with dance.

Researchers have begun to explore the processes by which dance produces positive outcomes for people with PD. For example, it has recently been proposed that motor-cognitive processes within dance, such as action observation, imitation and imagery, may contribute to positive motor and non-motor effects (Bek et al., 2020). These processes activate areas of the motor system to facilitate movement and 
learning (e.g., Abbruzzese et al., 2015), and motor simulation is also involved in social understanding (e.g., empathy) and interaction (lacoboni et al., 2005). Dance also provides other forms of internal and external cues, such as temporal patterning through music and rhythmic counting, that may facilitate movement for people with PD (Ashoori et al., 2015; de Dreu et al., 2011; Spaulding et al., 2013) and are often used within physiotherapy. These elements may differ between in-person, group contexts and individual, home-based participation.

The present study examined experiences and perceptions of digital dance programs among people with PD during the COVID-19 pandemic. The aims were to investigate engagement with these resources and to identify processes involved, potential benefits and advantages, as well as limitations of home-based dance.

\section{Methods}

In a collaboration between researchers and providers of the international community dance programs Dance for PD (Mark Morris Dance Group) and Silver Swans (Royal Academy of Dance), an online survey was designed to collect data from older adults with and without PD who were participating in or interested in home-based dance. To further ensure the relevance of the survey content, input into the design was also obtained from people with PD, dance instructors, and physiotherapists with experience in therapeutic dance settings.

A set of questions was compiled to investigate participants' experiences and views in the following areas: (i) access and usage, (ii) aspects of engagement and experience, (iii) advantages and disadvantages, and (iv) future participation. The majority of questions offered fixed-choice responses to minimise the need for participants to provide lengthy answers. Respondents not currently participating in at-home dance were directed to a subset of questions covering topics such as previous participation, barriers to accessing resources, and potential factors in selecting digital programs for future use.

The survey was created and administered using SelectSurvey.net (v4.033.002; ClassApps, Overland Park, KS, USA) and data collection took place from June to November 2020. The study was approved by the University of Manchester
Research Ethics Committee and all participants provided informed consent via an online form, completion of which was mandatory prior to entering the survey.

Potential respondents were contacted through mailing lists and newsletters of dance organisations and community groups. The survey was further advertised through a research volunteer list, social media and direct contact with dance practitioners known to the research team. In this paper we report the fixed-choice responses from people with PD; analysis of the full data set and open comments is ongoing and will be reported separately.

\section{Results}

Responses were collected from 276 people living with PD from 6 different countries; primarily the United Kingdom (68.1\%) and USA (26.4\%), with the remainder from Canada, Ireland, Switzerland and South Africa. Of these, 178 (75.8\% female; mean age 69.5 , range $47-88$ years) reported already using home-based dance programs, while 98 (73.5\% female; mean age 67.8 , range $45-85$ years) had not yet participated. Among those using at-home programs, $78.7 \%$ had previously attended inperson dance classes, while $50 \%$ of non-users had previously attended classes.

Summary statistics are presented in Table 1 and results are described under the subheadings below. Relationships between the number of perceived benefits, duration and frequency of use, previous participation, type of media used, and elements of engagement during practice were further analysed using independent t-tests and one-way ANOVAs followed up with Bonferronicorrected pairwise comparisons.

\section{Access and usage}

Respondents had been using home-based dance programs for periods ranging from a few weeks to several years, although $77 \%$ had started to use these directly in response to the COVID-19 restrictions. The majority were also using online platforms for other forms of exercise and leisure activities, as well as tools for social interaction such as social media sites, messaging applications and video calls.

Most participants (93.8\%) reported practicing dance at home at least once a week, with the largest proportion practicing more than twice 
weekly. Participants reported using a variety of media including live-streamed classes, interactive online classes, pre-recorded classes and DVDs. The majority had used more than one type of at-home dance resource, and of those using both live and recorded media, 64.5\% expressed a preference for live classes. Several different factors were rated as important when choosing classes, as noted in Table 1 , with the most frequently endorsed features
( $>50 \%$ of respondents) being dance style, familiarity of program/instructor and provision of scheduled classes.

The most commonly reported type of difficulty in accessing and using resources was connectivity or network problems, with smaller numbers reporting other issues. However, $61.8 \%$ of respondents did not experience any problems.

Table 1: Aspects of experience reported (\% respondents)

\begin{tabular}{ll}
\hline & Users \\
\hline Duration of use: & 29.2 \\
$0-3$ months & 26.4 \\
$3-6$ months & 18.5 \\
$6-12$ months & 16.9 \\
$>12$ months & 5.1 \\
\hline Frequency of use: & 29.2 \\
Less than once a week & 28.1 \\
Once a week & 36.5 \\
Twice a week & \\
More than twice a week & 66.3 \\
\hline Type of media/resource: & 47.2 \\
Live-streamed class & 69.1 \\
Interactive online class (e.g., Zoom) & 28.7 \\
Pre-recorded online class & 1.1 \\
DVD & \\
Other & \\
\hline Preferred format: &
\end{tabular}

Preferred format:

Live (including streamed, interactive)

Recorded (including online video, DVD)

Both live and recorded

No preference

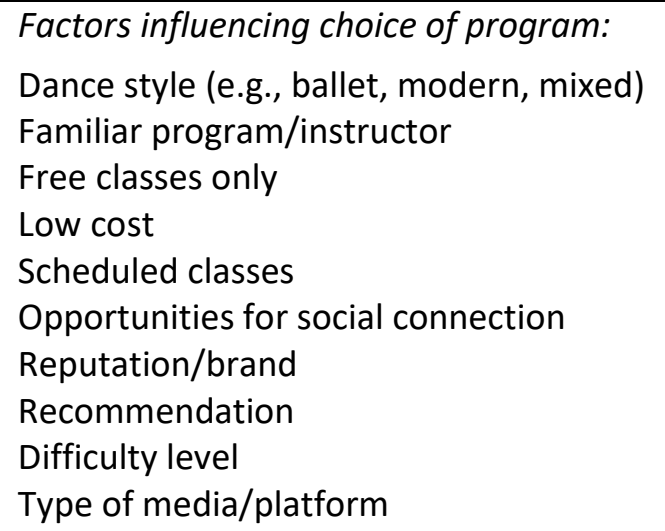

Type of media/platform

60.1 51.0 
Table 1 (cont.): Aspects of experience reported (\% respondents)

Use of other home-based resources

Other home-based activities

77.5

59.2

Online platforms/media for social interaction

73.6

71.4

Difficulties in accessing or using digital classes:

Connectivity/network problems

29.2

Setting up or using software

7.3

Image quality

6.7

Sound quality

15.2

No problems

61.8

\section{Elements of engagement:}

Watching the instructor closely

91.6

Vocalising the movements

23.6

Singing

42.1

Counting

44.4

Imagining how the movements would look if... (visual imagery)

26.4

Imagining how the movements would feel... (kinesthetic imagery)

18.5

Imagining moving like something else... (analogy / metaphor imagery)

26.4

Playing music in the background

4.5

Advantages of home-based dance:

Doesn't require travel

86.0

Flexible timing

60.7

Ease of access promotes more frequent participation

54.5

Maintains privacy

25.3

Ability to practice at own pace

42.1

Ability to express oneself without worrying about what others think

34.8

Disadvantages of home-based dance:

Reduced motivation without fixed routine

30.3

Difficulties in accessing or using technology

14.0

Lack of suitable space to practice

15.2

Lack of quiet time to practice

7.3

Absence of one-to-one support/tuition

25.3

Absence of social interaction

70.8

Aspects missed from in-person participation:

Interaction with the instructor

63.5

Interaction with others

70.8

42.9

Support/encouragement

38.8

33.7

Live music

31.5

13.3

Social activities before/after class

40.5

20.4

Note: percentages based on total number of participants providing a valid response to each item (i.e., excluding non-respondents) 


\section{Aspects of engagement and experience}

Most participants reported that they watched the instructor closely during classes. Auditory/rhythmic cues to support movement were engaged through counting, singing and vocalising the movements. Participants also endorsed all three types of imagery explored by the survey: visual (imagining the look of the movement), kinesthetic (imagining the feeling of the movement), and analogy/metaphor (imagining moving like something else such as an animal, a tree, waves on the ocean or falling leaves). Over a third of respondents reported using more than one type of imagery.

Perceived benefits of engaging with digital programs are presented in Figure 1 . These related to sensorimotor and functional abilities (ease of movement while dancing and in general; improved performance of everyday activities, balance, posture and rhythm) as well as cognitive/affective benefits (improved mood, confidence and concentration; reduced stress and anxiety). Improvements in energy and sleep quality were also reported. Very few participants (5.1\%) did not notice any benefits.

\section{Advantages and disadvantages}

Responses highlighted key advantages of homebased participation as not having to travel to a venue, choice of when to engage with the classes, and increased opportunities for frequent participation. Participants also appreciated the ability to practice at their own pace, express themselves freely, and maintain privacy.

The most frequently reported disadvantages were loss of social interaction, reduced motivation in the absence of a fixed routine, and absence of one-to-one support and tuition. Other disadvantages were not having a suitable space to practice, difficulties with technology, and a lack of quiet time for practice.

When compared to in-person classes, participants primarily reported missing the interaction with other dancers and the instructor, followed by social activities linked to classes, support and encouragement, and live music.

\section{Future participation}

Responses to questions about future participation in dance and different modes of delivery are illustrated in Figure 2. The greatest proportion of

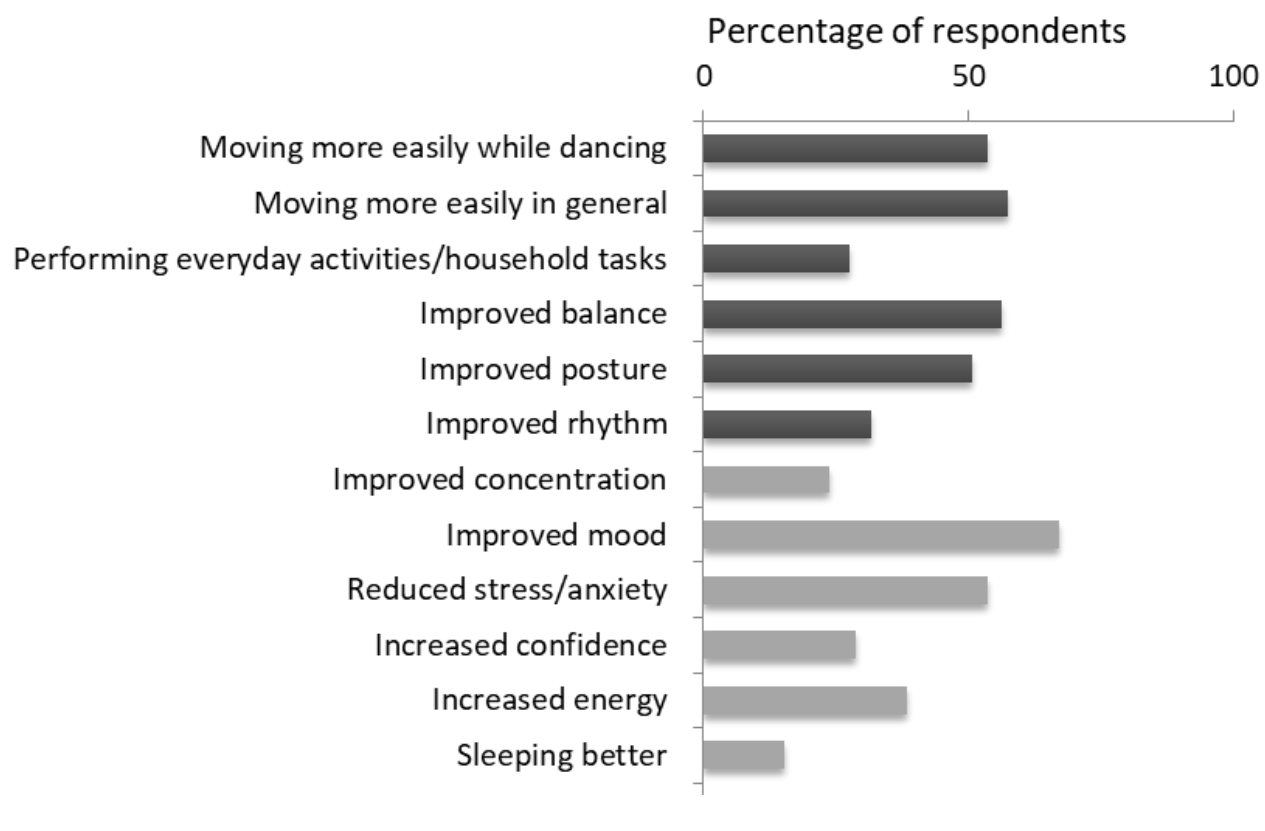

Figure 1. Perceived benefits of home-based dance illustrated by percentage of participants endorsing each outcome: Sensorimotor and functional benefits are indicated by the darker bars, followed by non-motor benefits. Only $5.1 \%$ of participants reported no benefits. 
respondents expressed a preference to continue with both in-person and digital classes if both options were available, rather than only one mode of participation. The majority of participants expressed an interest in receiving supplementary written and/or video-based educational resources to optimise the benefits of their dance practice, while a similar proportion were interested in trying out dance programs using an app and/or virtual reality.
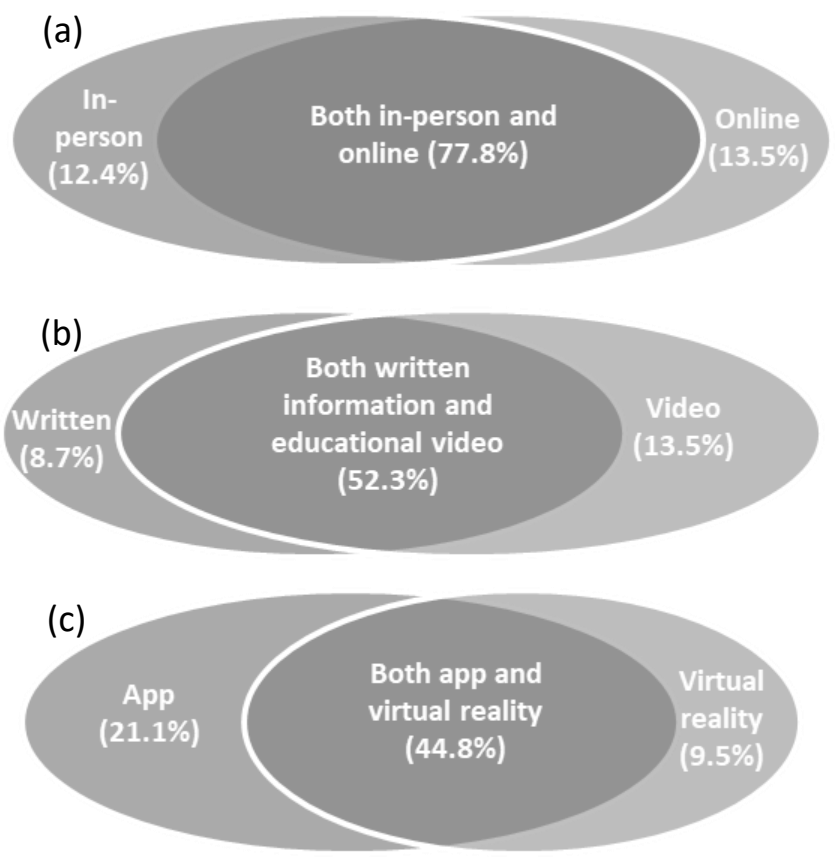

Figure 2. Preferences for future participation: Results indicated that the majority of respondents were interested in (a) continuing with both inperson and online dance classes, (b) receiving educational resources to optimise their dance practice, and (c) trying new modes of dance participation such as apps and virtual reality.

\section{Non-users}

Among respondents not currently using at-home dance programs, the primary barriers to participation were lack of knowledge about how to access the resources (33.9\%) and lack of motivation (30.4\%), with a small number of respondents citing lack of time (8.7\%), lack of interest $(0.9 \%)$, not having access to the internet or software required $(0.9 \%)$, or not expecting to benefit $(0.9 \%)$. As illustrated in Table 1, factors identified as important in choosing digital programs to use in future reflected those reported by current users, except that "familiar program/instructor" and "reputation/brand" were rated as less important.

\section{Relationships between participation, media type, elements and benefits}

There was a significant effect of duration of homebased practice on the number of perceived benefits $(F(3,155)=4.96 ; p=.003)$. Respondents using resources for more than 12 months reported more benefits than those participating for 0-3 months (mean difference $=2.04 ; p=.024), 3-6$ months (mean difference $=2.41 ; p=.006$ ) or $6-12$ months (mean difference $=2.62 ; p=.005$ ). There was also a significant effect of frequency of practice on the number of perceived benefits $(F(3,167)=3.79 ; p=.012)$, which was greater among those practicing more than twice a week than those practicing once a week (mean difference $=1.77 ; p=.014$ ). However, there was no difference in number of perceived benefits between respondents previously attending inperson classes and those not previously attending $(t(169)=.059 ; p=.95)$.

Media type significantly affected the number of perceived benefits $(F(2,166)=3.51 ; p=.032)$. Respondents using a combination of live and prerecorded classes reported a greater number of benefits than those only using pre-recorded materials (mean difference $=1.72 ; p=.039$ ).

As illustrated in Figure 3, greater numbers of benefits were reported by respondents who engaged in visual imagery $(\mathrm{t}(167)=2.69 ; \mathrm{p}=.008)$, kinesthetic imagery $(\mathrm{t}(167)=2.86 ; \mathrm{p}=.005)$ or analogy/metaphor imagery $(\mathrm{t}(167)=3.93 ; \mathrm{p}<.001)$ during classes than participants who did not use these elements. Singing during classes was also associated with a greater number of benefits $(t(170)=3.10 ; p=.002)$, but there were no significant differences for counting $(\mathrm{t}(170)=1.48 ; \mathrm{p}$ $=.14)$ or vocalising $(t(170)=1.92 ; p=.057)$. 


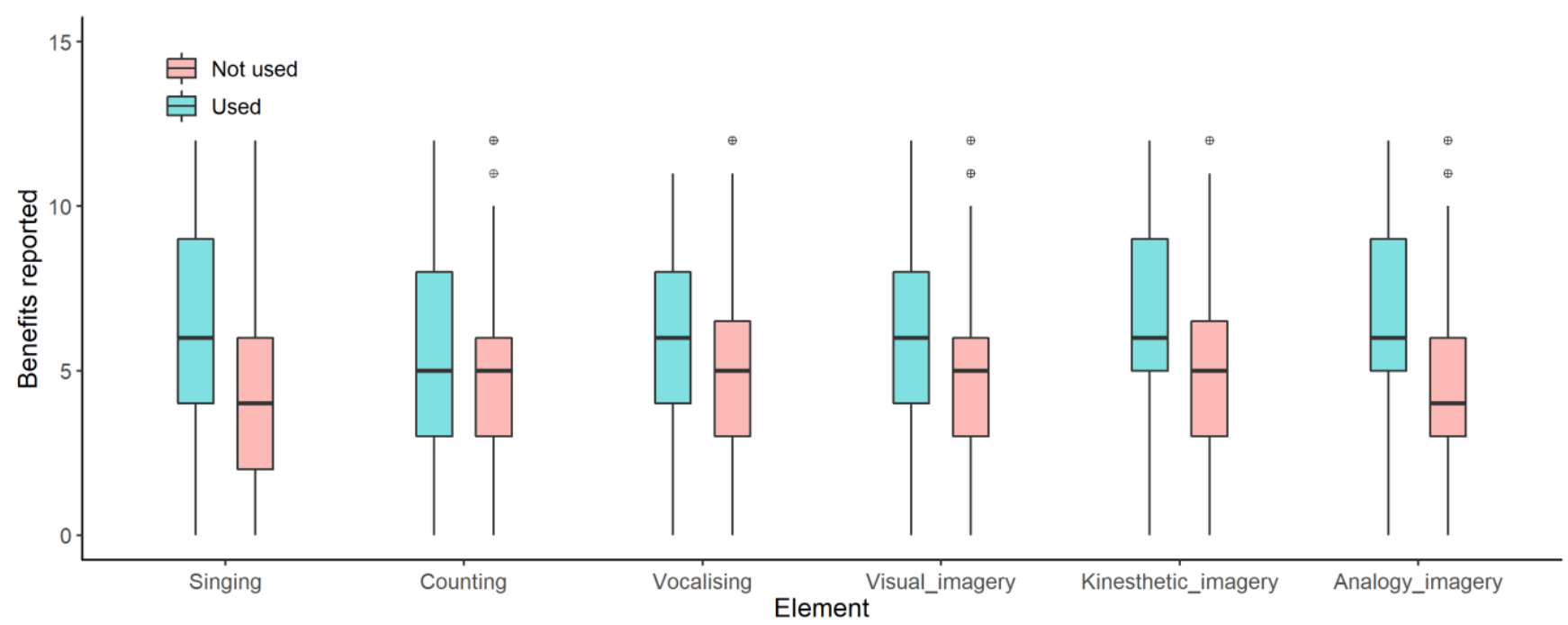

Figure 3. Self-reported benefits in relation to the use of different strategies and cues during home-based dance participation. Boxes show medians with quartiles and dots represent outliers. All three types of imagery (visual, kinesthetic and analogy/metaphor), as well as singing, were significantly associated with higher numbers of perceived benefits.

\section{Discussion}

This collaborative project investigated usage and experiences of home-based dance programs among people with PD, following the transition from in-person to online provision in response to the COVID-19 pandemic. The findings discussed below suggest potential avenues for future research into dance for people with PD, which will inform the development of new models of programming and provide further insights into the value of dance as a therapeutic option for PD.

The results of the survey indicated that people with PD were accessing and using a variety of home-based programs and resources, including interactive and streamed live classes, pre-recorded videos and DVDs. The majority of respondents had previously attended in-person classes and had begun to use digital classes as a direct consequence of COVID-19. Most respondents were practicing dance at least once a week, with many participating twice a week or more, suggesting a high level of motivation to continue dancing.

Despite concerns about technological barriers, very few participants experienced difficulties in accessing and setting up the required platforms, although connectivity problems were more widely reported. The majority of participants also reported using other home-based activity resources and social platforms, consistent with recent literature indicating the ability of people with PD to engage in online activities and therapeutic programs (Quinn et al., 2020; Schirinzi et al., 2020). However, around a third of respondents who were not currently using homebased programs reported a lack of knowledge of how to find and access them, which may include issues around familiarity with technology. It must also be acknowledged that the present study primarily reached individuals who were already engaged in digital technologies, with at least sufficient knowledge to use email and complete online surveys. To increase the accessibility of home-based programs, it will therefore be important to consider how to raise broader awareness and support participation among those who are not currently using the relevant technology. Additionally, the present findings predominantly reflect the experiences of participants in the UK and USA, and it will be important to understand the accessibility and potential benefits of home-based programs across diverse geographical and cultural settings.

Most participants had noticed some benefits of their home-based practice, with improvements reported across sensorimotor, functional, cognitive 
and affective domains, as well as in fatigue levels and sleep. These findings are consistent with reported benefits of in-person participation (Aguiar et al., 2016; dos Santos Delabary et al., 2018; Kalyani et al., 2019; McNeely et al., 2015; Shanahan, 2015; Sharp \& Hewitt, 2014). Greater numbers of reported benefits were associated with higher frequency of practice and longer length of at-home participation. However, the latter relationship was only found in those participating for more than 12 months, which may be attributable to those individuals previously using home-based resources alongside in-person classes, resulting in a higher level of overall experience. Nonetheless, the number of reported benefits did not differ according to whether or not respondents had previously attended in-person classes, indicating that positive effects may be obtained through home practice alone. Participants using a combination of live and pre-recorded materials reported more benefits than those using prerecorded videos only, which could reflect higher levels of motivation and engagement, although further research is needed to understand the effects of different media.

As dance practice involves motor-cognitive strategies and other cues that may positively impact outcomes such as movement and communication in people with PD (Bek et al., 2020), the present study examined the potential role of these in home-based participation. The use of different types of imagery (visual, kinesthetic and analogy/metaphor), as well as singing, were found to be associated with higher levels of perceived benefits. To further explore the significance of these elements, future research should examine their application in both individual/digital and group/in-person contexts. For example, imagery may be facilitated by live music and visual input from other dancers' movement during in-person participation (see Bek et al., 2020), whereas individuals may attend more closely to the instructor's movements, or more consciously utilise cognitive strategies or external cues, when outside of the group setting.

The present findings also reinforce the importance of social aspects of dance (Houston \& McGill, 2013; Kunkel et al., 2018). The lack of social interaction was the most frequently cited disadvantage of home-based practice and the most commonly missed aspect of in-person classes, followed by the absence of direct support from the instructor. Of those using different types of media for home practice, the majority of participants preferred live classes to recorded videos, possibly reflecting the higher level of interaction provided.

Given that digital resources are likely to remain an important component of provision going forward, a key finding is that the majority of respondents expressed interest in continuing with home practice alongside in-person classes. Respondents particularly appreciated the convenience and flexibility of home practice, and the frequency of participation may reflect the ease of access compared with travelling to classes, although the loss of routine was cited as a disadvantage of this mode of participation. It will be important to compare the outcomes of inperson and digital dance participation in future research. Qualitative analysis of open comments collected via the survey is ongoing and may reveal further insights into the nature of engagement and perceived benefits.

While it may not be possible to address all of the reported limitations of digital participation, such as not having suitable space at home for dancing, or the absence of live music, other features such as social interaction around class time and personalised support may be simulated or substituted to some extent. The longer-term benefits of at-home dance participation should also be investigated, particularly as motivation and engagement may decline over time, although this may be somewhat mitigated by providing regularly scheduled classes where possible.

Finally, many participants were interested in receiving educational materials to support their dance practice. This might include guidance on strategies to enhance engagement and potential benefits of dance, such as the internal and external cues noted above. Online peer support groups could also provide a forum for participants to share ideas on how to optimise their dance practice, while offering further opportunities for social interaction. A similar proportion of respondents were also open to trying new technologies such as an app or virtual reality. The feasibility of homebased exercise and dance programs using digital tools has been reported in people with PD (e.g., Allen et al., 2017; Song et al., 2018; Tunur et al., 2020) and, if found to enhance the benefits of dance, such approaches could be integrated into future programs, or offered as supplementary options to increase engagement. 


\section{Conclusions}

The present findings indicate that home-based dance programs are accessible and usable for people with $\mathrm{PD}$, while also identifying potential barriers to digital participation which should be addressed. The range of perceived benefits reflects previous findings from in-person programs, although further research is needed in order to understand how participation and outcomes may differ. There is a clear demand among current participants for continued use of digital resources alongside in-person provision, and these resources may also increase accessibility of dance and its therapeutic effects for people with PD who are unable to attend classes.

\section{Acknowledgements}

The authors wish to thank the following individuals for their input into the survey design: Aline Arakaki, Anne-Marie Booth, Gayathri Ganapathy, Helen Gould, Rachel Johnston, and Matthew Sullivan. We also thank Parkinson's UK and the Manchester Institute for Collaborative Research on Ageing for assistance with recruitment and sharing of the project.

\section{Disclosure statement}

Michelle Groves is a full-time employee of the Royal Academy of Dance and is involved in the development of its Silver Swans initiative. David Leventhal is a full-time employee of the Mark Morris Dance Group, which administers the Dance for $\mathrm{PD}^{\circledR}$ program. None of the authors have any other conflicts of interest.

\section{References}

Abbruzzese, G., Avanzino, L., Marchese, R. \& Pelosin, E. (2015). Action Observation and Motor Imagery: Innovative Cognitive Tools in the Rehabilitation of Parkinson's Disease. Parkinson's Disease. https://doi.org/10.1155/2015/124214

Aguiar, L. P. C., Da Rocha, P. A. \& Morris, M. (2016). Therapeutic dancing for Parkinson's disease. International Journal of Gerontology, 10(2), 64-70. https://doi.org/10.1016/j.ijge.2016.02.002

Allen, N. E., Song, J., Paul, S. S., Smith, S., O'Duffy, J., Schmidt, M., Love, R., Sherrington, C. \& Canning, C. G. (2017). An interactive videogame for arm and hand exercise in people with Parkinson's disease: A randomized controlled trial. Parkinsonism and Related Disorders. https://doi.org/10.1016/j.parkreldis.2017.05. 011

Ashoori, A., Eagleman, D. M. \& Jankovic, J. (2015). Effects of auditory rhythm and music on gait disturbances in Parkinson's disease. In Frontiers in Neurology. https://doi.org/10.3389/fneur.2015.00234

Bek, J., Arakaki, A. I., Lawrence, A., Sullivan, M., Ganapathy, G. \& Poliakoff, E. (2020). Dance and Parkinson's: A review and exploration of the role of cognitive representations of action. In Neuroscience and Biobehavioral Reviews. https://doi.org/10.1016/j.neubiorev.2019.12. 023

Brown, E. G., Chahine, L. M., Goldman, S. M., Korell, M., Mann, E., Kinel, D. R., Arnedo, V., Marek, K. L. \& Tanner, C. M. (2020). The Effect of the COVID-19 Pandemic on People with Parkinson's Disease. Journal of Parkinson's Disease. https://doi.org/10.3233/JPD-202249

Chaudhuri, K. R., Healy, D. G. \& Schapira, A. H. V. (2006). Non-motor symptoms of Parkinson's disease: Diagnosis and management. In Lancet Neurology. https://doi.org/10.1016/S14744422(06)70373-8

Cilia, R., Bonvegna, S., Straccia, G., Andreasi, N. G., Elia, A. E., Romito, L. M., Devigili, G., Cereda, E. \& Eleopra, R. (2020). Effects of COVID-19 on Parkinson's Disease Clinical Features: A Community-Based Case-Control Study. Movement Disorders. https://doi.org/10.1002/mds.28170

de Dreu, M. J., Kwakkel, G., van Wegen, E. E. H., Poppe, E. \& van der Wilk, A. S. D. (2011). Rehabilitation, exercise therapy and music in patients with Parkinson's disease: a metaanalysis of the effects of music-based movement therapy on walking ability, balance and quality of life. Parkinsonism \& Related Disorders, 18, S114-S119. https://doi.org/10.1016/s1353- 
8020(11)70036-0

Dorsey, E.R., Elbaz, A., Nichols, E., Abd-Allah, F., Abdelalim, A., Adsuar, J. C., Ansha, M. G., Brayne, C., Choi, J. Y. J., Collado-Mateo, D., Dahodwala, N., Do, H. P., Edessa, D., Endres, M., Fereshtehnejad, S. M., Foreman, K. J., Gankpe, F. G., Gupta, R., Hankey, G. J., ... Murray, C. J. L. (2018). Global, regional, and national burden of Parkinson's disease, 19902016: a systematic analysis for the Global Burden of Disease Study 2016. The Lancet Neurology. https://doi.org/10.1016/S14744422(18)30295-3

dos Santos Delabary, M., Komeroski, I. G., Monteiro, E. P., Costa, R. R. \& Haas, A. N. (2018). Effects of dance practice on functional mobility, motor symptoms and quality of life in people with Parkinson's disease: a systematic review with meta-analysis. In Aging Clinical and Experimental Research. https://doi.org/10.1007/s40520-017-0836-2

Helmich, R. C. \& Bloem, B. R. (2020). The Impact of the COVID-19 Pandemic on Parkinson's Disease: Hidden Sorrows and Emerging Opportunities. In Journal of Parkinson's Disease. https://doi.org/10.3233/JPD-202038

Houston, S. \& McGill, A. (2013). A mixed-methods study into ballet for people living with Parkinson's. Arts \& Health, 5(2), 103-119. https://doi.org/10.1080/17533015.2012.7455 80

lacoboni, M., Molnar-Szakacs, I., Gallese, V., Buccino, G., Mazziotta, J. C. \& Rizzolatti, G. (2005). Grasping the intentions of others with one's own mirror neuron system. Plos Biology, 3(3), 529-535. https://doi.org/10.1371/journal.pbio.003007 9

Kalyani, H. H. N., Sullivan, K., Moyle, G., Brauer, S., Jeffrey, E. R., Roeder, L., Berndt, S. \& Kerr, G. (2019). Effects of Dance on Gait, Cognition, and Dual-Tasking in Parkinson's Disease: A Systematic Review and Meta-Analysis. In Journal of Parkinson's Disease. https://doi.org/10.3233/JPD-181516

Keus, S., Munneke, M., Graziano, M., Paltamaa, J., Pelosin, E., Domingos, J., Bruhlmann, S., Ramaswamy, B., Prins, J., Struiksma, C., Rochester, L., Nieuwboer, A. \& Bloem, B. (2014). European physiotherapy guideline for Parkinson's disease: Development \& implementation. Movement Disorders, 29,
S537-S537.

Kunkel, D., Robison, J., Fitton, C., Hulbert, S., Roberts, L., Wiles, R., Pickering, R., Roberts, H. \& Ashburn, A. (2018). It takes two: the influence of dance partners on the perceived enjoyment and benefits during participation in partnered ballroom dance classes for people with Parkinson's. Disability and Rehabilitation, 40(16). https://doi.org/10.1080/09638288.2017.1323 029

McNeely, M. E., Duncan, R. P. \& Earhart, G. M. (2015). Impacts of dance on non-motor symptoms, participation, and quality of life in Parkinson disease and healthy older adults. Maturitas, 82(4), 336-341. https://doi.org/10.1016/j.maturitas.2015.08. 002

Quinn, L., MacPherson, C., Long, K. \& Shah, H. (2020). Promoting physical activity via telehealth in people with parkinson disease: The path forward after the COVID-19 pandemic? Physical Therapy. https://doi.org/10.1093/ptj/pzaa128

Schirinzi, T., Di Lazzaro, G., Salimei, C., Cerroni, R., Liguori, C., Scalise, S., Alwardat, M., Mercuri, N. B., Pierantozzi, M., Stefani, A. \& Pisani, A. (2020). Physical Activity Changes and Correlate Effects in Patients with Parkinson's Disease during COVID-19 Lockdown. Movement Disorders Clinical Practice. https://doi.org/10.1002/mdc3.13026

Shanahan. (2015). Dance for People With Parkinson Disease: What Is the Evidence Telling Us? (vol 96, pg 141, 2015). Archives of Physical Medicine and Rehabilitation, 96(10), 1931.

https://doi.org/10.1016/j.apmr.2015.08.420

Sharp, K. \& Hewitt, J. (2014). Dance as an intervention for people with Parkinson's disease: A systematic review and metaanalysis. Neuroscience and Biobehavioral Reviews, 47(September), 445-456. https://doi.org/10.1016/j.neubiorev.2014.09. 009

Song, J., Paul, S. S., Caetano, M. J. D., Smith, S., Dibble, L. E., Love, R., Schoene, D., Menant, J. C., Sherrington, C., Lord, S. R., Canning, C. G. \& Allen, N. E. (2018). Home-based step training using videogame technology in people with Parkinson's disease: a singleblinded randomised controlled trial. Clinical 
Rehabilitation.

https://doi.org/10.1177/0269215517721593

Spaulding, S. J., Barber, B., Colby, M., Cormack, B., Mick, T. \& Jenkins, M. E. (2013). Cueing and Gait Improvement Among People With Parkinson's Disease: A Meta-Analysis. Archives of Physical Medicine and Rehabilitation, 94(3), 562-570.

https://doi.org/10.1016/j.apmr.2012.10.026

Subramanian, I. (2020). Virtual Parkinson's Disease Support Groups in the COVID-19 Era: Social Connection in the Time of Social Distancing. In Movement Disorders Clinical Practice. https://doi.org/10.1002/mdc3.12994

Tunur, T., DeBlois, A., Yates-Horton, E., Rickford, K. \& Columna, L. A. (2020). Augmented realitybased dance intervention for individuals with Parkinson's disease: A pilot study. Disability and Health Journal.

https://doi.org/10.1016/j.dhjo.2019.100848 